\title{
Using the Saphenous Artery and Great Saphenous Vein Combined with Anterolateral Thigh Flap to Treat Skin Defects after Amputation
}

\author{
Ling Dong Kong, MD ${ }^{1, *}$ Han Xiong Cheng, $\mathrm{MD}^{1, *}$ Tao Nie, MD ${ }^{1}$ Min Dai, MD \\ ${ }^{1}$ Department of Orthopedics, The First Affiliated Hospital of \\ Nanchang University, Nanchang, Jiangxi, People's Republic of China \\ Address for correspondence Tao Nie, MD, Department of \\ Orthopedics, The First Affiliated Hospital of Nanchang University, \\ Nanchang, Jiangxi 330000, People's Republic of China \\ J Reconstr Microsurg Open 2018;3:e70-e73. \\ (e-mail: ncnietao@163.com).
}

\author{
Abstract \\ Keywords \\ - saphenous artery \\ - great saphenous vein \\ - anterolateral thigh \\ flap \\ - amputation
}

Background Aim of this study was to determine the feasibility of using the saphenous artery (SA) and great saphenous vein (GSV) as recipient vessels, combined with anterolateral thigh (ALT) flap, in the treatment of skin defects after lower limb amputation. Methods From June 2015 to June 2017, 12 patients (average age, 33.5 years; range, 14-56 years; males, 9; female, 3) with large skin defects and symptoms of bone exposure in the proximal lower extremity were included in our study. The patients underwent emergency treatment and multiple debridement combined with vacuum sealing drainage therapy, followed by free flap surgery using the SA and GSV as recipient vessels, and ALT to cover the wound.

Results All 12 patients who underwent free flap surgery survived, but two patients had distal flap necrosis, which, however, was salvaged with conservative measures. All patients were satisfied with the postoperative outcome at the 3 and 6-month follow-up. Conclusion The SA and GSV can be used as recipient vessels, combined with ALT, to treat skin defects after lower limb amputation.
With the development in modern and transportation industries, severe lower limb injuries caused by high-energy accidents are becoming more common in clinical practice. Patients who experience such accidents often have severe nerve and blood vessel damage ${ }^{1,2}$ with some necessitating amputation. To preserve the knee joint, many doctors often perform proximal lower limb amputation ${ }^{3}$ leaving behind a large area of soft tissue defects as well as bone exposure in the lower limb. To cover the remaining wounds, free flap graft is often necessary. ${ }^{4-7}$ However, fewer blood vessels that can be used for anastomosis are left, and inflammatory reaction after trauma can occur. Thus, choosing suitable blood vessels to anastomose with the descending branch of the contralateral circumflex femoral artery is critical to the success of the treatment. The objective of this study was to

* Ling Dong Kong and Han Xiong Cheng are co-first authors. determine the feasibility of using the saphenous artery (SA) and great saphenous vein (GSV) as recipient vessels combined with anterolateral thigh (ALT) flap in the treatment to skin defects after lower limb amputation.

\section{Methods}

\section{Patient Information}

This study included 12 patients (male, 9; female, 3; average age, 33.5 years; range, $14-56$ years) who underwent treatment (left leg, 9 cases; right leg, 3 cases) at our institution due to traffic accident injuries from June 2015 to June 2017. Based on the Gustilo's classification, there were 8 Gustilo's IIIB and 4 Gustilo's IIIC type cases, all accompanied by comminuted fracture of the tibia and fibula. All 12 patients underwent emergency debridement, vascular anastomosis, external stenting, and vacuum sealing drainage (VSD) treatment. During the operation of one

Copyright $\odot 2018$ by Thieme Medical Publishers, Inc., 333 Seventh Avenue, New York, NY 10001, USA. Tel: +1(212) 584-4662.

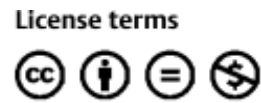

(c) 1 License terms $\$$ received

May 24, 2018

accepted after revision

August 30, 2018
DOI https://doi.org/

10.1055/s-0038-1675407. ISSN 2377-0813. 
patient, we found sever vascular injury accompanied by a long defect; hence, we performed emergency amputation and VSD treatment. Another patient had high fever and vascular crisis on the second day after operation. Emergency surgery was performed, and we noted infection and thus performed amputation. To prevent postoperative infection, all patients underwent free flap graft after multiple debridement and VSD. Free flaps were used to repair wounds 16 to 36 days after injury (-Fig. 1).

\section{Surgical Method}

The patients underwent multiple debridement and systemic supportive treatment before surgery to correct anemia, improve hypoalbuminemia, correct electrolyte disturbances, and improve general condition (-Fig. 1A). When the granulation tissue of the wound became red, indicative of controlled infection, and there was no obvious symptom of infection, the wound was repaired with ALT with the patient placed in supine position; continuous epidural or hard-waist combined block anesthesia is used in such cases and the position need not be changed during surgery. First, the size of the soft tissue defect in the lower limb was assessed using the template, and the flap of the same size was determined in the lateral femoral area to ensure that the soft tissue of the defect could be fully covered (-Fig. 1B). Second, knee medial incision was made, entering from the gap between the sartorius and gracilis muscles, pulling the sartorius muscle outward which revealed the SA, saphenous nerve, and GSV. Then the SA, along with the GSV was used as recipient vessel and anastomosed to the descending branch of the circumflex femoral artery and vein, respectively (-Fig. 1C). The lateral femoral cutaneous nerve was anastomosed to the saphenous nerve (-Fig. 1D).

\section{Postoperative treatment}

A drainage tube was placed under the flap after operation, and it was removed 2 to 3 days later. Continuous irradiation of the lamp, conventional application of anti-infective, anticoagulant, and antivasospasm drug treatment, and close observation of the flap's temperature, color, and capillary filling reaction was performed (Fig. 1 E, F).

\section{Results}

All patients were admitted to the hospital because of trauma. The lower limbs were severely damaged and they had to be amputated. Patients'data are presented in - Table 1. However, all of the 12 free flaps survived; 10 of them had primary healing, but distal flap necrosis appeared in 2 cases. Fortunately, it was salvaged with conservative measures. After 3 to 6-month follow-up in all cases, the flap was free from bloated appearance and had fine texture. The soft tissue defect was repaired satisfactorily and all skin grafts survived in receiving area. Based on follow-up observations, we found that the patients were satisfied with the treatment. After the prosthesis was installed, the ability to walk was restored and many patients returned to work and activities of daily living.

\section{Discussion}

All 12 patients in this study required lower limb amputations due to serious traffic accidents. Considering that the patients are young and have higher functionality and aesthetic requirements, we had to operate relatively far away from the knee to preserve the function of the knee joint as much as

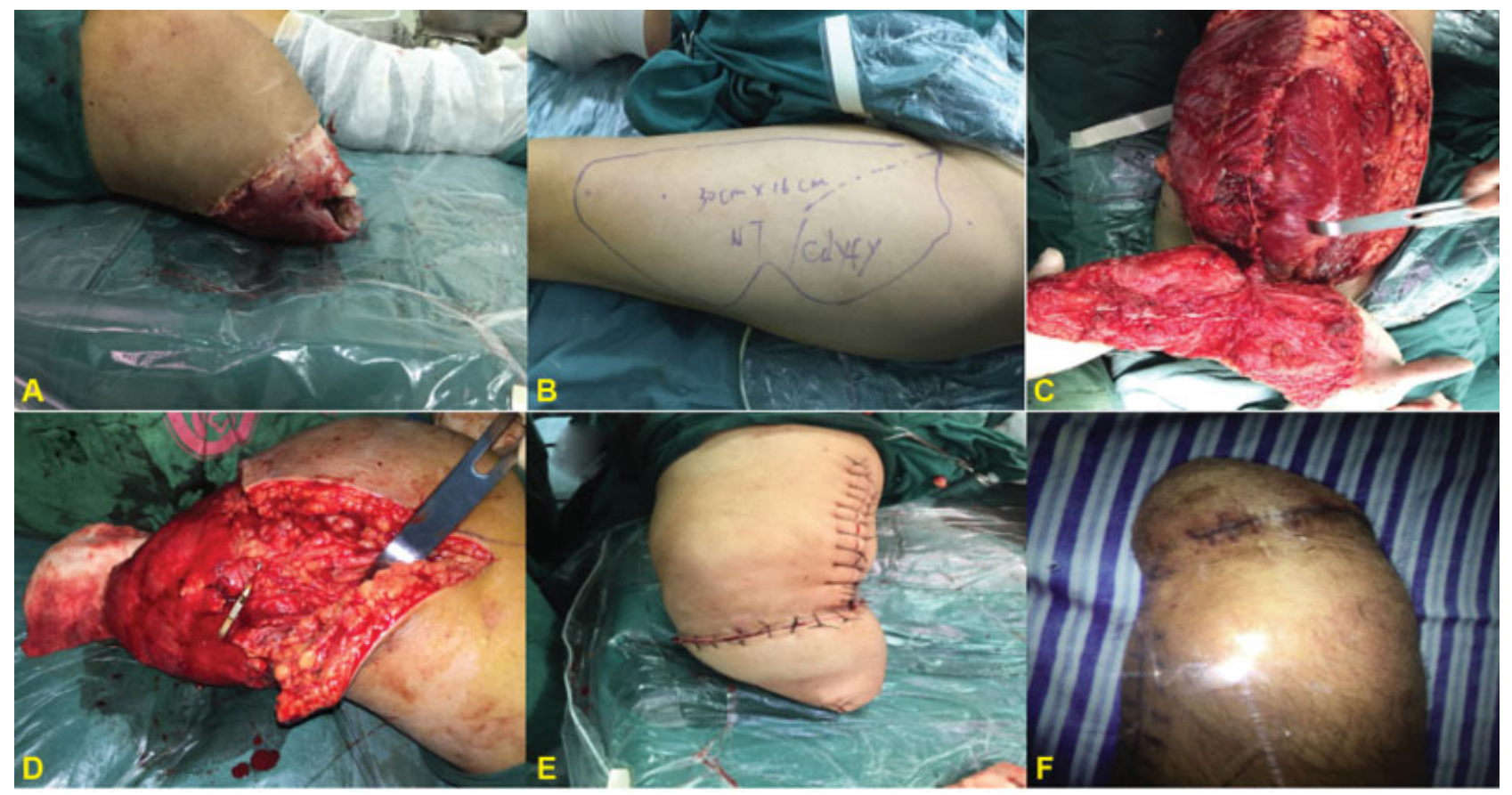

Fig. 1 A 42-year-old man was referred for treatment of a mutilating crush injury on the right lower limb, after three sessions of vacuum-assisted suctioning and debridement, the patient received a $16 \times 30 \mathrm{~cm}^{2}$ free flap with no complications and recovered in 3 months. (A) After three times of vacuum-assisted suctioning and debridement. (B) Design of the free flap. (C) Reveal the contralateral descending branch of the circumflex femoral artery and flap preparation before transplantation. (D) Reveal the saphenous artery, great saphenous vein, and saphenous nerve. (E) Postoperative appearance. (F) At 3 months after surgery, the flap intact without complications. 
Table 1 Patients' information

\begin{tabular}{|l|l|l|l|l|l|l|l|}
\hline Case & Sex & Age (y) & Side & Gustilo & Flap size $\left.\mathbf{( c m}^{2}\right)$ & Complication & Outcome \\
\hline 1 & M & 42 & R & IIIC & $30 \times 16$ & N/A & Survival \\
\hline 2 & F & 27 & L & IIIB & $32 \times 18$ & Distal necrosis & Survival \\
\hline 3 & M & 35 & L & IIIB & $34 \times 15$ & N/A & Survival \\
\hline 4 & M & 49 & R & IIIC & $28 \times 17$ & N/A & Survival \\
\hline 5 & M & 56 & L & IIIB & $29 \times 18$ & N/A & Survival \\
\hline 6 & F & 14 & L & IIIC & $26 \times 14$ & Distal necrosis & Survival \\
\hline 7 & M & 24 & L & IIIB & $28 \times 17$ & N/A & Survival \\
\hline 8 & M & 37 & L & IIIB & $27 \times 16$ & N/A & Survival \\
\hline 9 & M & 32 & L & IIIB & $33 \times 15$ & N/A & Survival \\
\hline 10 & F & 31 & L & IIIC & $38 \times 19$ & N/A & Survival \\
\hline 11 & M & 27 & R & IIIB & $34 \times 14$ & N/A & Survival \\
\hline 12 & M & 28 & L & IIIB & $27 \times 18$ & N/A & Survival \\
\hline
\end{tabular}

Abbreviations: F, female; L, left; M, Male; N/A, not applicable; R, right.

possible; in other words, the flexion and extension of the knee joint had to be maintained after installing an artificial limb in the future. However, there are contradictions. If we lowered the area of amputation, the risk of infection increases. Moreover, the bone was largely exposed which made it difficult to close the soft tissue wound. This was one of the key aspects to be considered.

The knee has very delicate structure and complex function $^{8,9}$ and the appearance requirements are also high. If we proceeded with the adjacent flap transplantation, the coverage area would not have been enough due to the limited area of the flap and the short pedicle and the contraction of the scar would likely affect the function of the knee joint. ${ }^{10,11}$ Cross-leg flap transplantation has also been reported ${ }^{12,13}$ but this method requires the patient to have a high degree of compliance; the patient must maintain the same posture for a long time and the patients often cannot tolerate this, so we choose free flap for transplantation. In 12 patients in the present study, the SA and GSV were used as the recipient blood vessels, combined with the ALT to repair the skin and soft tissue defects under the knee joint after amputation. This surgical method is particularly suitable in the following situations: (1) anterior tibial artery or posterior tibial artery is injured; (2) long-term pathological changes in the anterior tibial artery or posterior tibial artery have occurred; (3) presence of inflammation scars or ulcers in the anterior tibial artery or the posterior tibial artery; (4) the anterior tibial artery or the posterior tibial artery is not suitable for exploration and anastomosis. ${ }^{14,15}$ In addition, it is not wise to perform anastomosis to the descending branch of the circumflex femoral artery using major blood vessels as the recipient vessels because distal limb blood flow is very poor after trauma. If this is done, it will increase the possibility of distal ischemic necrosis.

The position of the SA is relatively stable with occasional variations. ${ }^{16}$ We can easily find it; moreover, we identified and located the artery by preoperative Doppler's imaging. ${ }^{17}$ Through clinical observation, we found that the caliber of the SA and the descending branch of circumflex femoral artery were equivalent, making it easier to anastomose; moreover, the blood flow is relatively stable and can provide sufficient flow for the flap. The return flow of the GSV is also sufficient and the survival rate of the flap is greatly improved. ${ }^{18}$ The anastomosis of the saphenous nerve and the ALT nerve can keep the sense of the knee. Another advantage is that the size and thickness of the ALT can meet the soft tissue requirements around the knee joint.

For patients who need surgery, the wound must be aseptic ${ }^{19}$; hence, we ensure that the patient has no fever, the C-reactive protein level and erythrocyte sedimentation rate are normal and bacterial culture results are negative. We use Doppler to identify that there is no embolization of the GSV and no venous damage before operation. ${ }^{20}$ Postoperative anticoagulation therapy and antivascular spasm treatment are conventionally used; furthermore, continuous irradiation of light is also necessary to increase the blood flow of blood vessels. All patients were evaluated preoperatively for physical condition ${ }^{21}$ and surgery was performed by an experienced physician.

However, this surgical method also has limitations. When the skin defect around the knee is large, the skin defect at the donor site may not be able to be sutured. Generally, we perform skin transplantation to cover the wound. However, Tong et al suggested that transplantation with free groin flaps is better. ${ }^{22}$ Another concern of this design is the prolonged operative time because microsurgery procedures are intensive and require longer operation time. However, if two surgical teams are available, it can shorten the operation time.

\section{Conclusion}

The surgical approach of using the SA and GSV as recipient blood vessels combined with contralateral ALT flap to repair large areas of the skin and soft tissue defects in the proximal leg is simple and safe, has a high survival rate, and can meet knee wound coverage requirements. ${ }^{19,23}$ The biggest advantage of 
this surgery is the retention of function of the knee. Hence, we highly recommend its clinical application.

\section{Conflict of Interest}

None.

\section{References}

1 MacKenzie EJ, Bosse MJ, Kellam JF, et al. Characterization of patients with high-energy lower extremity trauma. J Orthop Trauma 2000;14(07):455-466

2 Bibbo C, Nelson J, Fischer JP, et al. Lower extremity limb salvage after trauma: versatility of the anterolateral thigh free flap. J Orthop Trauma 2015;29(12):563-568

3 Wong $\mathrm{CH}$, Ong YS, Wei FC. The anterolateral thigh-Vastus lateralis conjoint flap for complex defects of the lower limb. J Plast Reconstr Aesthet Surg 2012;65(02):235-239

$4 \mathrm{Xu} \mathrm{ZF}$, Sun CF, Duan WY, et al. Clinical anatomical study and evaluation of the use of the free anteromedial thigh perforator flaps in reconstructions of the head and neck. Br J Oral Maxillofac Surg 2013;51(08):725-730

5 Gong ZJ, Zhang S, Ren ZH, Zhu ZF, Liu JB, Wu HJ. Application of anteromedial thigh flap for the reconstruction of oral and maxillofacial defects. J Oral Maxillofac Surg 2014;72(06):1212-1225

6 Hupkens P, Van Loon B, Lauret GJ, et al. Anteromedial thigh flaps: an anatomical study to localize and classify anteromedial thigh perforators. Microsurgery 2010;30(01):43-49

7 Cigna E, Chen HC, Ozkan O, Sorvillo V, Maruccia M, Ribuffo D. The anteromedial thigh free flap anatomy: a clinical, anatomical, and cadaveric study. Plast Reconstr Surg 2014;133(02):420-429

8 Meland NB, Fisher J, Irons GB, Wood MB, Cooney WP. Experience with 80 rectus abdominis free-tissue transfers. Plast Reconstr Surg 1989;83(03):481-487

9 Ao M, Nagase Y, Mae O, Namba Y. Reconstruction of posttraumatic defects of the foot by flow-through anterolateral or anteromedial thigh flaps with preservation of posterior tibial vessels. Ann Plast Surg 1997;38(06):598-603

10 Hong JP. The use of supermicrosurgery in lower extremity reconstruction: the next step in evolution. Plast Reconstr Surg 2009; 123(01):230-235
11 Choi DH, Goh T, Cho JY, Hong JP. Thin superficial circumflex iliac artery perforator flap and supermicrosurgery technique for face reconstruction. J Craniofac Surg 2014;25(06):2130-2133

12 Zenn MR, Levin LS. Microvascular reconstruction of the lower extremity. Semin Surg Oncol 2000;19(03):272-281

13 Fischer JP, Wink JD, Nelson JA, et al. A retrospective review of outcomes and flap selection in free tissue transfers for complex lower extremity reconstruction. J Reconstr Microsurg 2013;29 (06):407-416

14 Hill JB, Vogel JE, Sexton KW, Guillamondegui OD, Corral GA, Shack $\mathrm{RB}$. Re-evaluating the paradigm of early free flap coverage in lower extremity trauma. Microsurgery 2013;33(01):9-13

15 Kimura N, Saito M, Sumiya Y, Itoh N. Reconstruction of hand skin defects by microdissected mini anterolataral thigh perforator flaps. J Plast Reconstr Aesthet Surg 2008;61(09):1073-1077

16 Bosse MJ, McCarthy ML, Jones AL, et al; Lower Extremity Assessment Project (LEAP) Study Group. The insensate foot following severe lower extremity trauma: an indication for amputation? J Bone Joint Surg Am 2005;87(12):2601-2608

17 Yuan F, Chung KC. Impact of safety net hospitals in the care of the hand-injured patient: a national perspective. Plast Reconstr Surg 2016;138(02):429-434

18 Lin YT, Lin CH, Wei FC. More degrees of freedom by using chimeric concept in the applications of anterolateral thigh flap. J Plast Reconstr Aesthet Surg 2006;59(06):622-627

19 Yu P. Inverse relationship of the anterolateral and anteromedial thigh flap perforator anatomy. J Reconstr Microsurg 2014;30(07): 463-468

20 Lakhiani C, Lee MR, Saint-Cyr M. Vascular anatomy of the anterolateral thigh flap: a systematic review. Plast Reconstr Surg 2012;130(06):1254-1268

21 Chana JS, Wei FC. A review of the advantages of the anterolateral thigh flap in head and neck reconstruction. Br J Plast Surg 2004;57 (07):603-609

22 Tong D, Liu Y, Wu LW, Zhu S, Zhu J, Chen S. Free groin flap for aesthetic and functional donor-site closure of the anterolateral thigh flap. J Plast Reconstr Aesthet Surg 2016;69(08): $1116-1120$

23 Kang MJ, Chung CH, Chang YJ, Kim KH. Reconstruction of the lower extremity using free flaps. Arch Plast Surg 2013;40(05): 575-583 